\title{
Efecto de las Bajas Concentraciones de Nitratos y Fosfatos sobre la Acumulación de Astaxantina en Haematococcus pluvialis UTEX 2505
}

\author{
Alejandra M. Miranda ${ }^{1}$, Edgar A. Ossa ${ }^{1}$, Gabriel J. Vargas² y Alex A. Sáez ${ }^{1 *}$ \\ (1) Escuela de Ingeniería, Univ. EAFIT, Cra. 49, Medellín-Colombia. \\ (e-mail: ammirandap@eafit.edu.co; eossa@eafit.edu.co; asaez@eafit.edu.co \\ (2) I\&D Cementos Argos S.A, Centro de Argos para la Innovación, Cra. 49, Medellín-Colombia. \\ (e-mail: eossa@eafit.edu.co) \\ * Autor a quien va dirigido la correspondencia
}

Recibido May. 14, 2018; Aceptado Jul. 17, 2018; Versión final Ago. 17, 2018, Publicado Feb. 2019

\begin{abstract}
Resumen
En este trabajo se evalúa el efecto de la concentración de nitrógeno y fósforo sobre la acumulación de Astaxantina en Haematococcus pluvialis UTEX 2505, utilizando un diseño de experimentos factorial $3^{2}$. Las variables de respuestas cuantificadas fueron el crecimiento celular, la producción de Astaxantina, perfil lipídico y el módulo de Young de la membrana celular. Se encontró que la productividad celular aumenta al incrementar los niveles de nitrógeno, en contraste con las bajas concentraciones de nitrógeno y fósforo que muestra un efecto positivo sobre la producción de Astaxantina. El estrés generado por la limitación de nutrientes (fuente de nitrógeno y fósforo) disminuye la rigidez de la pared celular en la microalga. Como conclusión, para obtener concentraciones más altas de Astaxantina, es necesario limitar los niveles de nitrógeno y fósforo.
\end{abstract}

\section{Effect of the Low Concentration of Nitrates and Phosphates on the Accumulation of Astaxantin in Haematococcus pluvialis UTEX 2505}

\begin{abstract}
In this study, the effect of the concentration of nitrogen and phosphorus on the accumulation of Astaxanthin in Haematococcus pluvialis UTEX 2505 was evaluated using a factorial $3^{2}$ design. The dependent variables were cell growth, Astaxanthin production, lipid profile and Young's modulus of the cell membrane. It was found that cellular productivity increases when nitrogen levels were high, in contrast to low concentrations of nitrogen and phosphorus that showed a positive effect on Astaxanthin production. The stress generated by the source of nutrients decreases the rigidity of the cellular wall in the microalgae. As a conclusion, to obtain higher concentrations of Astaxanthin it is necessary to limit the nitrogen and phosphorus levels.
\end{abstract}

Keywords: Astaxanthin; Lipids; Antioxidant; Haematococcus pluvialis; Young's module 


\section{INTRODUCCIÓN}

En la actualidad la salud de los seres humanos se ha visto afectada por diferentes tipos de enfermedades, entre las que se encuentran las llamadas crónico-degenerativas (diabetes, cáncer, patologías cardiovasculares cardiopatías). Un proceso que lleva consigo la formación de estas enfermedades es la producción de radicales libres, (Justo y Gutiérrez, 2002) estos pueden ser producto de factores como la exposición a radiaciones ionizantes, aditivos químicos en alimentos, herbicidas, contaminación ambiental, mala alimentación, entre otros. (Maldonado et al., 2010).

El cuerpo humano posee mecanismos de defensa contra el estrés oxidativo (antioxidantes endógenos) inducido por los radicales libres (Valko et al., 2017), donde la función es prevenir o retardar el proceso de oxidación que deterioran la célula; estas moléculas son conocidas como antioxidantes. Sin embargo, debido al aumento de los factores externos que originan la formación de estas especies reactivas se ha generado un desequilibrio, llevando a la necesidad de incluir en la dieta suplementos alimenticios que contengan antioxidantes para prevenir diversos cambios fisiológicos que puedan provocar destrucción celular (San Miguel y Gil, 2009). En la naturaleza existen diversas moléculas con alto poder antioxioxidante entre las que se encuentran la vitamina $\mathrm{E}$, vitamina $\mathrm{C}$, flavonoides, resveratrol, ácido ascórbico, compuestos fenólicos, tocoferol, carotenoides entre otros (Yamashita, 2013; Comert y Gokmen, 2018). Entre estos existe el pigmento astaxantina que es considerado como uno de los más importantes en la salud humana debido a que presenta propiedades farmacológicas, antiinflamatorias, alto poder antioxidante actividad anticancerígena, efecto antimicrobiano contra el Helicobacter pylori. (Hernández et al., 2015; Galarza et al., 2018).

La Astaxantina es un carotenoide que pertenece al grupo de las xantofilas, posee 40 carbonos en su estructura molecular $\left(\mathrm{C}_{40} \mathrm{H}_{52} \mathrm{O}_{4}\right)$, su nombre científico es 3,3'-dihidroxi- $\beta$, $\beta^{\prime}$-caroteno-4,4'-diona. Posee una cadena polienica compuesta por 13 enlaces dobles conjugados alternados con enlaces simples que le proporcionan a la molécula una alta capacidad antioxidante y le brindan el característico color de rojo intenso (Camacho et al., 2013; Zuluaga et al., 2018). Este carotenoide se puede obtener a partir de síntesis química (Higuera et al., 2006) o de forma natural mediante la biosíntesis - mevalónica de diversos microorganismos como las levaduras Xanthophyllomyces dendrorhous (Zheng et al., 2006), Phaffia rhodozyma (Colabella y Libkind, 2016), microalgas como Dunaliella salina (Anila et al., 2016), Chlorella zofingiensis (Jin et al., 2014) y Haematococcus pluvialis (Camacho et al., 2013), siendo esta ultima el microorganismo que produce mayor cantidad del antioxidante.

Haematococcus pluvialis es una microalga verde unicelular Chlorophyceae de agua dulce perteneciente al orden Volvocales y familia Haematococcaceae. H. pluvialis es conocida como productora de Astaxantina al ser sometida a diferentes condiciones de estrés (alta intensidad lumínica, deficiencia de nutrientes, altas concentraciones de sal entre otros) (Orosa et al., 2005). La síntesis de novo de la Astaxantina tiene lugar en el citosol, acumulándose como pequeñas gotas en el citoplasma (cuerpos lipídicos citosólicos) (Galarza et al., 2018). La síntesis se lleva a cabo a partir de la ruta de los terpenoides o isoprenoides (producto de la vía mevalónica) por intermedio del acetato proveniente de la respiración y fotosíntesis, la cual generalmente incluye cinco pasos: (i) formación del isopentenil difosfato, (ii) condensación por etapas de isopreno hasta llegar a la formación del fitoeno, (iii) serie de reacciones de desaturación para llegar a la formación del licopeno, (iv) reacciones de ciclación que generan el $\beta$-caroteno y finalmente (v) la hidroxilación que da como origen la Astaxantina (Shah et al., 2016; Chen et al., 2015).

Para aumentar la síntesis de Astaxantina en la microalga se han realizado varios estudios donde se evalúa el efecto de diferentes factores sobre la acumulación de este metabolito secundario. Orosa et al. (2005), cultivaron a $\mathrm{H}$. pluvialis en diferentes concentraciones de $\mathrm{NaNO}_{3}$ para determinar el efecto sobre el crecimiento celular y la acumulación de Astaxantina, indicando que la concentración óptima de nitrato para obtener Astaxantina y para evitar el cese de la división celular es de $0.15 \mathrm{~g} / \mathrm{L}$ de $\mathrm{NaNO}_{3}$. De igual manera Choi et al. (2011), luego de realizar estudios donde variaron la intensidad lumínica, reportó que la máxima concentración de Astaxantina (26 mg/L) se obtiene a $500 \mu \mathrm{mol} / \mathrm{m}^{2} \mathrm{~s}$. Del mismo modo, Giannelli et al. (2015) evaluaron la influencia de la temperatura en la producción de Astaxantina estableciendo a una temperatura de alrededor los $30^{\circ} \mathrm{C}$ se inhibe la división celular y se induce la síntesis de Astaxantina, aumentando así el contenido de 10 a 15 veces comparado con el cultivo a condiciones óptimas.

Debido a la diversidad de industrias basadas en microalgas (suplementos dietéticos, cosméticos, productos farmacéuticos y biocombustibles) y en busca de mejorar la obtención de estos productos, se han realizado estudios de propiedades mecánicas como resistencia a la tracción, fuerza adhesiva, viscoelasticidad o módulo de Young (propiedad importante para la lisis celular) (Warren et al., 2014), mediante el uso de equipos que permiten alta resolución en imágenes y medidas de fuerza como lo es el microscopio de fuerza atómica (AFM). EI AFM puede trabajar con muestras biologías tanto en ambientes secos como acuosos, facilita el cálculo del módulo de Young y permite una caracterización mecánica de las células. En la actualidad no se han reportado 
estudios científicos acerca del efecto de diferentes factores sobre de la resistencia de la célula de $H$. pluvialis y la relación que esta presenta con otros productos de interés que sintetiza la microalga. El objetivo de este trabajo consistió en evaluar el efecto de la concentración de nitrógeno y fósforo sobre el crecimiento celular, producción de Astaxantina, acumulación de lípidos y módulo de Young en H. pluvialis UTEX 2505 cultivada en fotobiorreactores (FBR) tubulares de 20 litros bajo condiciones naturales de temperatura, iluminación y suministro de dióxido de carbono.

\section{METODOLOGÍA}

Haematococcus pluvialis UTEX 2505 fue obtenida de la UTEX (The Culture Collection of Algae), y es perteneciente al cepario del laboratorio de biotecnología del centro de Argos para la innovación (CAPI) ubicado dentro de la universidad EAFIT sede Medellín - Colombia. Para definir el medio de cultivo de la microalga en FBR de $20 \mathrm{~L}$ se utilizó como base el medio Basal Bold modificado (BBM-Modificado) (Cifuentes et al., 2003). Para este trabajo se dejaron constantes las siguientes composición del medio: $20.0 \mathrm{~g} / \mathrm{L}$ de $\mathrm{CaCl}_{2} \cdot 2 \mathrm{H}_{2} \mathrm{O}, 7.5 \mathrm{~g} / \mathrm{L}$ de $\mathrm{MgSO}_{4} \cdot 7 \mathrm{H}_{2} \mathrm{O}, 2.5 \mathrm{~g} / \mathrm{L}$ de NaCl, $10.0 \mathrm{~g} / \mathrm{L}$ EDTA Na $2,4.9 \mathrm{~g} / \mathrm{L} \mathrm{FeSO} 4,11.5 \mathrm{~g} / \mathrm{L} \mathrm{H}_{3} \mathrm{BO}_{4}$, $8.8 \mathrm{~g} / \mathrm{L} \mathrm{ZnSO} 4,1.4 \mathrm{~g} / \mathrm{L} \mathrm{MnCl}_{2} \cdot 4 \mathrm{H}_{2} \mathrm{O}, 0.7 \mathrm{~g} / \mathrm{L} \mathrm{MoO}, 1.5 \mathrm{~g} / \mathrm{L} \mathrm{CuSO} 4 \cdot 5 \mathrm{H}_{2} \mathrm{O}, 0.5 \mathrm{~g} / \mathrm{L}\left(\mathrm{NO}_{3}\right)_{2} \mathrm{Co}_{3} 6 \mathrm{H}_{2} \mathrm{O}(\mathrm{Cifuentes}$ et al., 2003). Ensayos preliminares mostraron muy baja concentración celular en tratamientos con $0.15 \mathrm{~g} / \mathrm{L}$ de nitratos en el medio de cultivo, razón por la cual para los diferentes tratamientos se modificó la concentración de las fuentes de nitrógeno $\left(\mathrm{NaNO}_{3}\right)$ y de fósforo $\left(\mathrm{KH}_{2} \mathrm{PO}_{4}\right.$ y $\left.\mathrm{K}_{2} \mathrm{HPO}_{4}\right)$ entre los niveles 1.0 - 0.6 - $0.2 \mathrm{~g} / \mathrm{L}$ para $\mathrm{NaNO}_{3}$ (Orosa et al., 2005; Zhang et al., 2014) y $1.0-0.6-0.2$ g/L para $\mathrm{K}_{2} \mathrm{HPO}_{4}$ y $\mathrm{KH}_{2} \mathrm{PO}_{4}$ (Borowitzka et al., 1991).

\section{Propagación de células.}

La cepa $H$. pluvialis UTEX 2505 perteneciente al cepario del laboratorio de Biotecnología del centro de Argos para la Innovación, se encontraba crioconservada a $-80^{\circ} \mathrm{C}$ utilizando como preservante el glicerol, esta fue descongelada en baño serológico de agua (Memmert, WNB 7-45 Alemania) a $35^{\circ} \mathrm{C}$ durante 90 segundos. La cepa fue repicada en Agar-BBM entre $\mathrm{pH} 6.4-6.6$ y se conservó a temperatura ambiente. Posteriormente a volúmenes de $50 \mathrm{~mL}$ de medio de cultivo estéril BBM-liquido (cultivo de activación) se adicionó toda la biomasa microalgal presente en las cajas de Petri, seguido con la etapa del preinóculo que contenía $860 \mathrm{~mL}$ de medio de cultivo estéril BBM-liquido con $100 \mathrm{~mL}$ de suspensión algal proveniente del cultivo de activación. Las condiciones empleadas fueron: temperatura $25 \pm 5^{\circ} \mathrm{C}$, fotoperiodo $12: 12$, intensidad de luz $52 \mu \mathrm{mol} / \mathrm{m}^{2} \mathrm{~s}$ y burbujeo de aire enriquecido con $3 \%$ de $\mathrm{CO}_{2}$. La preparación del inoculo se desarrolló en FBR cilíndricos de $20 \mathrm{~L}$, elaborados en acrílico transparente, la dispersión de los gases en el medio se realizó mediante una membrana difusora ubicada en la parte inferior del FBR y las condiciones de crecimiento fueron temperatura ambiente, fotoperiodos naturales, flujo de aire de $2.5 \mathrm{Lpm}$, burbujeo de aire enriquecido con $3 \%$ de $\mathrm{CO}_{2}$, medio de cultivo BBM-liquido no esterilizado y concentración celular inicial $0.2 \mathrm{~g} / \mathrm{L}$. El sistema experimental está conformado por 30 FBR (incluyendo los FBR empleados en la preparación del inoculo), distribuidos en 5 grupos de 6 FBR de $20 \mathrm{~L}$ cada uno. Todos los ensayos se realizaron bajo condiciones naturales de luz y temperatura, con una inyección de aire enriquecido con $3 \%$ de $\mathrm{CO}_{2}$ y concentración celular inicial de $0.2 \mathrm{~g} / \mathrm{L}$. La determinación de Astaxantina y colecta del cultivo se realizó al final de la fase exponencial.

\section{Cinética de crecimiento celular y determinación de parámetros.}

El crecimiento celular fue cuantificado mediante el método de peso seco cada 48 horas, hasta alcanzar la fase estacionaria, utilizando filtros estériles de éster de celulosa (Advantec MFS, Inc) con poro de $0.45 \mu \mathrm{m}$, diámetro de $47 \mathrm{~mm}$ y balanzas de humedad Sartorius Mark-3. Para determinar los parámetros cinéticos no estructurados y no segregados (Exponencial) se utilizaron valores promedio de los resultados experimentales obtenidos de la fermentación en batch. La ecuación (1) describe el modelo exponencial, en el cual $\mu$ max representa la tasa máxima de crecimiento específico de células de microalga (días ${ }^{-1}$ ), y X es la concentración celular.

$$
\frac{d X}{d t}=\mu_{\max } X
$$

\section{Cuantificación de metabolitos.}

La cuantificación de Astaxantina se realizó por triplicado usando el método sugerido por Zhang et al. (2009) con DMSO para la extracción. En la cuantificación se utilizó un espectrofotómetro ultravioleta visible con longitud de onda de $492 \mathrm{~nm}$. El contenido de Astaxantina se obtuvo mediante la ecuación (2), para la cual Ast es la cantidad de Astaxantina en $\mu \mathrm{g} / \mathrm{mL}, A_{492}$ es la absorbancia medida a $492 \mathrm{~nm}, 10^{6}$ el factor para expresar los carotenoides en $\mu \mathrm{g}, \mathrm{V}$ el volumen del solvente utilizado en $\mathrm{mL}, \mathrm{E}^{1 \%}{ }_{1 \mathrm{~cm}}$ representa el coeficiente de extinción 
específico para la Astaxantina en $\mu \mathrm{g} / \mathrm{mL}$ y 100 el coeficiente para eliminar el factor de porcentaje.

$$
\mathrm{Ast}=\frac{\ddot{\mathrm{A}}_{472} * 10^{6} * \mathrm{~V}}{\mathrm{E}_{1 \mathrm{~cm}}^{1 \%} * 100}
$$

En cuanto la cuantificación de ésteres metílicos de ácidos grasos extraídos de $H$. pluvialis UTEX 2505, se empleó un cromatógrafo de gases marca Shimadzu modelo GC 2014, con columna RT-2560 con espesor de la película de $0.20 \mu \mathrm{m}$, longitud de la columna de $100 \mathrm{~m}$ con diámetro interno de $0.25 \mathrm{~mm}$, el modo de inyección fue split, y la cantidad de volumen inyectado fue de $1 \mu \mathrm{L}$.

\section{Determinación del módulo de Young.}

El AFM permite medir la fuerza generada entre el material a ser evaluado y una punta afilada sujeta a un cantiléver, el cual sondea la superficie de la muestra mientras se registran los datos (Howland y Benatar, 2000). Para la cuantificación del módulo de Young de H. pluvialis UTEX 2505, se utilizó un microscopio de fuerza atómica marca Park Systems, Modelo XE7, con cantiléver B50-NCHR y fuerza aplicada de $28 \mathrm{~N} / \mathrm{m}$.

\section{Diseño de experimentos.}

Para determinar el efecto de bajas concentraciones de la fuente de nitrógeno $\left(\mathrm{NaNO}_{3}\right)$ y fósforo $\left(\mathrm{KH}_{2} \mathrm{PO}_{4}\right.$ $\mathrm{K}_{2} \mathrm{HPO}_{4}$ ) en el crecimiento celular, producción de Astaxantina, acumulación de lípidos y módulo de Young de H. pluvialis UTEX 2505, se utilizó un diseño factorial $3^{2}$ evaluando tres niveles $(1.0-0.6-0.2 \mathrm{~g} / \mathrm{L})$ y dos factores (fuente de nitrógeno y fósforo), realizando tres réplicas por tratamiento dando un total de 27 tratamientos experimentales.

El análisis estadístico de los resultados se realizó por medio de un análisis de varianza (ANOVA) con el 95\% de confiabilidad, usando el Software estadístico STATGRAPHICS Centurión versión XVI, en el cual se comprobaron los supuestos del ANOVA mediante test cuantitativos de: Shapiro- Wilk, Kolmogorv, Levene y Durbin - Watson. Se utilizó el test de Tukey para establecer que tratamientos presentaron efecto sobre las variables de respuestas, las barras de error presentadas en las gráficas corresponden al error, para cada uno de los tratamientos.

\section{RESULTADOS Y DISCUSIÓN}

H. pluvialis UTEX 2505 creció en los diferentes tratamientos del medio de cultivo BBM- modificado a diferentes concentraciones de la fuente de nitrógeno $\left(\mathrm{NaNO}_{3}\right)$ y fósforo $\left(\mathrm{KH}_{2} \mathrm{PO}_{4}\right.$ y $\left.\mathrm{K}_{2} \mathrm{HPO}_{4}\right)$, presentando un día de fase de adaptación, seguido de la fase exponencial la cual terminó entre el octavo y décimo primer día de cultivo. La mayor concentración celular obtenida fue para los tratamientos que contenían niveles de las fuentes de nitrógeno y fósforo más alto $(1.0 \mathrm{~g} / \mathrm{L})$, mostrando valores entre $1.5-1.9 \mathrm{~g} / \mathrm{L}$; a diferencia con los tratamientos donde la concentración de los factores era menor $(0.2 \mathrm{~g} / \mathrm{L})$, los cuales arrojaron valores de concentración celular de $0.85-1.0 \mathrm{~g} / \mathrm{L}$. Los parámetros cinéticos calculados $t_{d}$ y $\mu$ ( $t_{d}$ : tiempo de duplicación y $\mu_{x}$ : velocidad especifica de crecimiento) para el modelo exponencial son presentados en la tabla 1. Los resultados obtenidos en cuanto a la velocidad especifica de crecimiento máxima son comparables con los reportados por Orosa et al. (2005), los cuales oscilaron entre $(0.12-0.46)$ días $^{-1}$, sugiriendo que la concentración de nitrógeno en el medio de cultivo juega un papel importante en la tasa de división celular.

Tabla 1: Parámetros cinéticos de H. pluvialis UTEX 2505 en diferentes concentraciones de la fuente de nitrógeno y fósforo.

\begin{tabular}{|c|c|c|c|c|c|c|}
\hline \multirow[b]{2}{*}{ Nomenclatura } & \multirow[b]{2}{*}{$\begin{array}{l}\text { Nitrógeno } \\
(g / L)\end{array}$} & \multirow[b]{2}{*}{$\begin{array}{c}\text { Fósforo } \\
(g / L)\end{array}$} & \multicolumn{4}{|c|}{ Modelo exponencial } \\
\hline & & & $\begin{array}{c}\mu \\
\left(d^{-1}\right)\end{array}$ & $\begin{array}{c}t d \\
\text { (días) }\end{array}$ & $\begin{array}{c}X_{0} \\
(g / L)\end{array}$ & $R^{2}$ \\
\hline $\mathrm{N}_{1} \mathrm{P}_{1}$ & 1.0 & 1.0 & $0.196 \pm 0.021$ & $3.525 \pm 0.357$ & $0.226 \pm 0.028$ & $0.979 \pm 0.075$ \\
\hline $\mathrm{N}_{1} \mathrm{P}_{0}$ & 1.0 & 0.6 & $0.179 \pm 0.031$ & $3.855 \pm 0.053$ & $0.262 \pm 0.013$ & $0.974 \pm 0.095$ \\
\hline $\mathrm{N}_{1} \mathrm{P}_{-1}$ & 1.0 & 0.2 & $0.171 \pm 0.010$ & $4.034 \pm 0.249$ & $0.259 \pm 0.088$ & $0.970 \pm 0.045$ \\
\hline $\mathrm{N}_{0} \mathrm{P}_{1}$ & 0.6 & 1.0 & $0.143 \pm 0.015$ & $4.840 \pm 0.441$ & $0.246 \pm 0.091$ & $0.972 \pm 0.091$ \\
\hline $\mathrm{N}_{0} \mathrm{P}_{0}$ & 0.6 & 0.6 & $0.153 \pm 0.017$ & $4.506 \pm 0.495$ & $0.240 \pm 0.059$ & $0.977 \pm 0.030$ \\
\hline $\mathrm{N}_{0} \mathrm{P}_{-1}$ & 0.6 & 0.2 & $0.200 \pm 0.004$ & $3.455 \pm 0.079$ & $0.243 \pm 0.066$ & $0.953 \pm 0.125$ \\
\hline $\mathrm{N}_{-1} \mathrm{P}_{1}$ & 0.2 & 1.0 & $0.194 \pm 0.039$ & $3.565 \pm 0.899$ & $0.200 \pm 0.081$ & $0.929 \pm 0.116$ \\
\hline $\mathrm{N}_{-1} \mathrm{P}_{0}$ & 0.2 & 0.6 & $0.214 \pm 0.015$ & $3.237 \pm 0.226$ & $0.193 \pm 0.096$ & $0.947 \pm 0.082$ \\
\hline$N_{-1} P_{-1}$ & 0.2 & 0.2 & $0.176 \pm 0.090$ & $3.936 \pm 0.102$ & $0.250 \pm 0.077$ & $0.933 \pm 0.042$ \\
\hline
\end{tabular}


Al evaluar de manera independiente el efecto causado de los factores sobre las variables dependientes, concentración celular final $(x)$ y velocidad especifica de crecimiento $\left(\mu_{\max }\right)$, mediante un análisis de varianza (ANOVA) no se encontraron diferencias significativas entre los tratamientos $(P>0.05)$ para ambas variables, lo que indica que no hay incidencia en el cambio de los niveles sobre las dos variables dependientes. Li et al. (2015) afirman que el nitrógeno es un componente principal de las proteínas y los ácidos nucleicos favoreciendo el crecimiento celular. Aunque el nitrógeno y el fósforo participan de la misma forma en la constitución de las moléculas orgánicas fundamentales, los resultados no evidencian efecto positivo como podría esperarse, lo que puede ser ocasionado por el orden de magnitud de las variables de respuesta y el error propio de los ensayos.

Los resultados de productividad presentados en la figura 1, se analizaron mediante un análisis de varianza (ANOVA), donde se encontraron diferencias significativas $(p<0.05)$ entre los tratamientos con un nivel de confianza del $95 \%$. Para establecer las diferencias entre los tratamientos respecto a la productividad, se utilizó el test de rangos múltiples Tukey. Los tratamientos donde la fuente de nitrógeno $\left(\mathrm{NaNO}_{3}\right)$ fue de $1.0 \mathrm{~g} / \mathrm{L}$ presentaron mayor productividad, lo que sugiere que la productividad es mayor cuando la fuente de nitrógeno aumenta. Este comportamiento posiblemente pueda explicarse por la mayor disponibilidad de nitrógeno para la formación de biomoléculas utilizadas en la multiplicación celular. Resultados similares reporto (Zhang et al., 2014; Hernández et al., 2015), afirmando que el incremento en la concentración de nitrógeno en el medio de cultivo, genera un aumento en la productividad de biomasa.

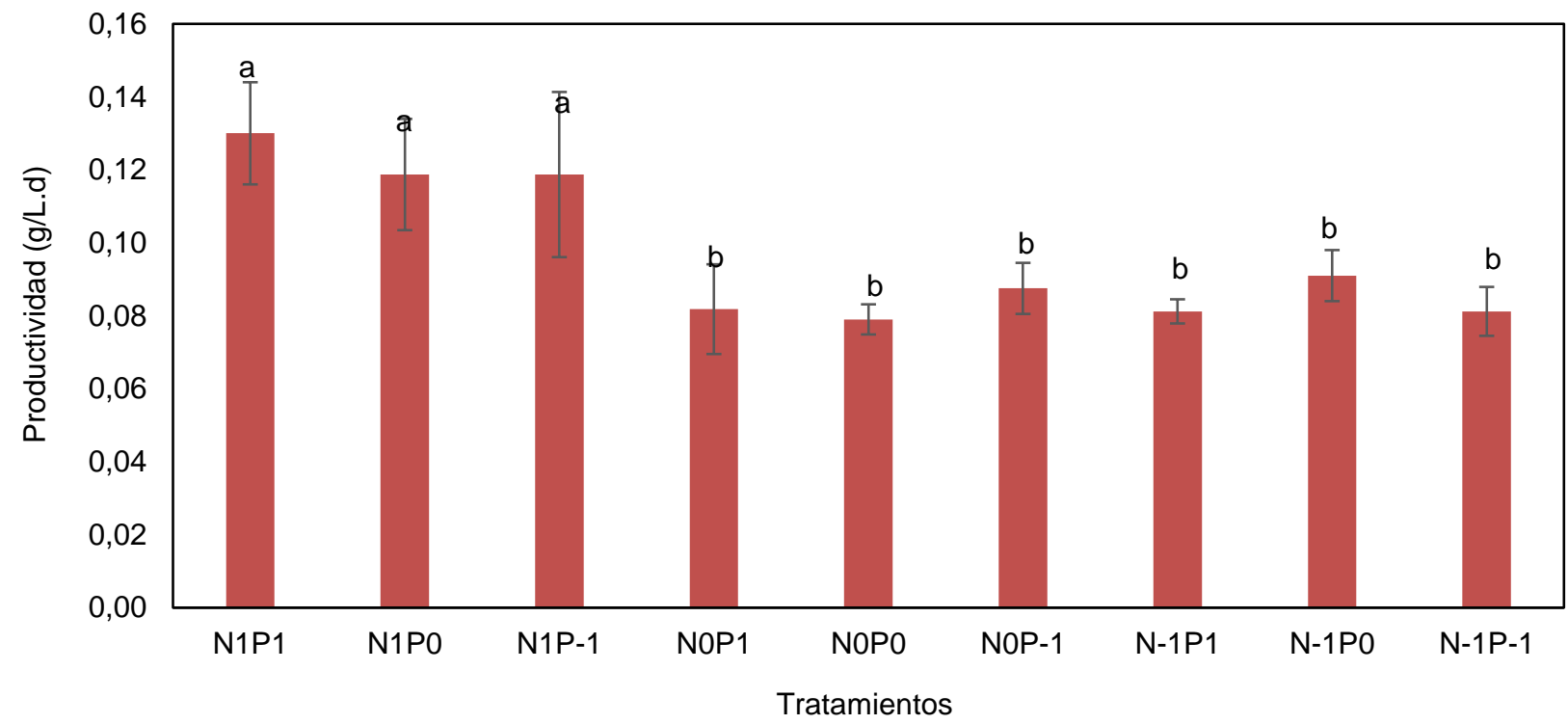

Fig. 1: Productividad de biomasa obtenida de $H$. pluvialis UTEX 2505 en diferentes concentraciones de la fuente de nitrógeno y fósforo. Las letras iguales, corresponden a grupos estadísticamente homogéneos según el test de Tukey, con un nivel de confianza del 95\%

Los resultados obtenidos en cuanto a la interacción causada por los factores sobre la acumulación de Astaxantina en $H$. pluvialis UTEX 2505 se presentan en la figura 2, determinando que la interacción fue positiva a niveles bajos de nitrógeno $(0.2 \mathrm{~g} / \mathrm{L})$. Este resultado sugiere que a bajas concentraciones en la fuente de nitrógeno se estimula la síntesis del carotenoide, a diferencia del efecto causado de las bajas concentraciones de fósforo, el cual ejerce un efecto negativo en la síntesis de Astaxantina, es decir, bajas concentraciones de la fuente de fósforo generan una disminución en la acumulación de Astaxantina en la microalga.

El efecto de la interacción de la fuente de nitrógeno y la fuente de fósforo sobre la concentración de Astaxantina se observa en la figura 3, con una máxima acumulación $(168.4 \mu \mathrm{g} / \mathrm{mL})$ del carotenoide en el tratamiento $\mathrm{N}_{-1} \mathrm{P}_{0}$, en contraste con el tratamiento $\mathrm{N}_{1} \mathrm{P}_{1}$, el cual presentó el valor más bajo $(41.9 \mu \mathrm{g} / \mathrm{mL})$. Lo anterior indica que para aumentar la acumulación de Astaxantina en la microalga es recomendable bajas concentraciones en la fuente de nitrógeno y altas concentraciones de fósforo, resaltando que la inanición de nitrógeno estimula la síntesis de Astaxantina, originando que la célula se encuentre en el estado aplanospóra. Investigaciones realizadas por Suh et al. (2006) encontraron que para obtener altas producciones de Astaxantina $(357 \mathrm{mg} / \mathrm{L})$, es necesario generar estrés a la célula por medio de la inanición de nitrógeno (Zhang et al., 2015). 


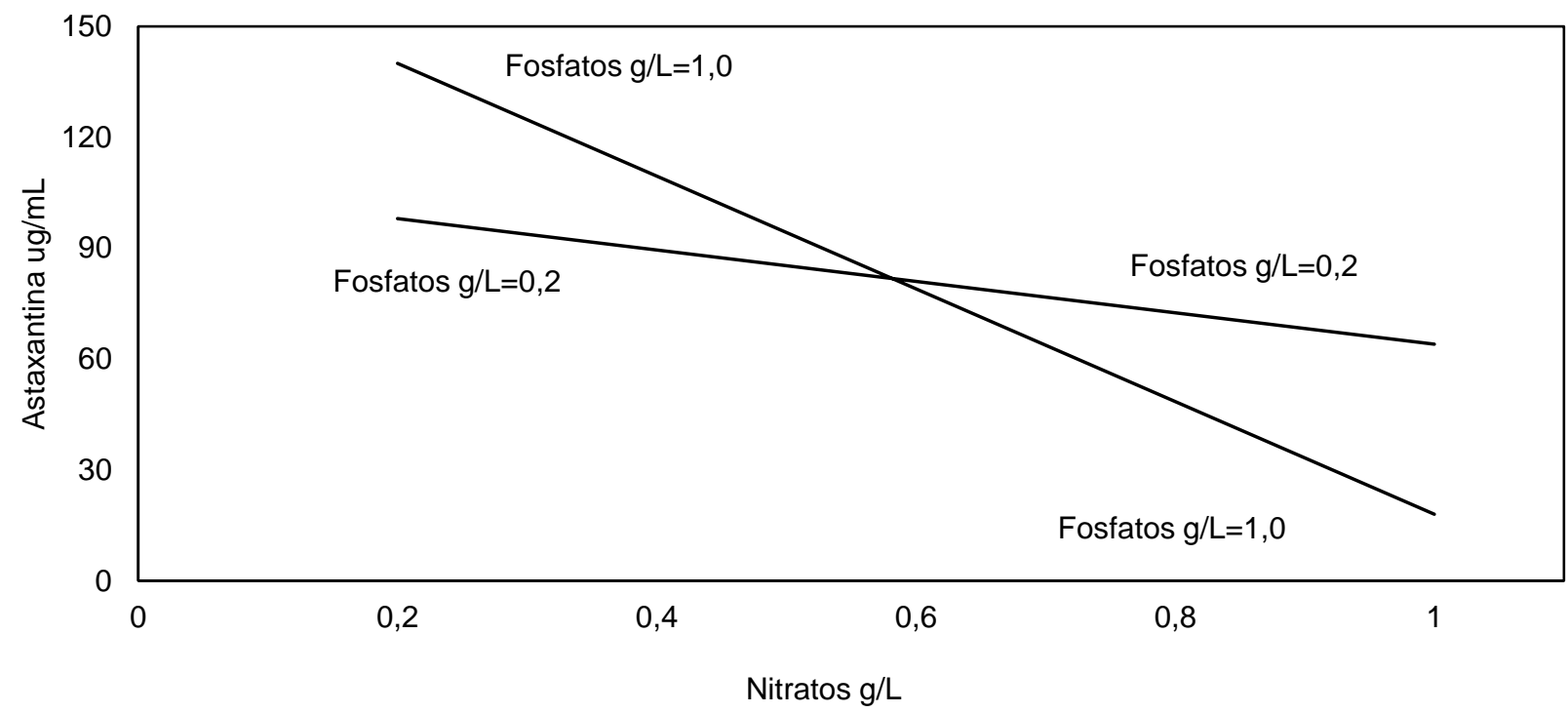

Fig. 2: Efecto de las interacciones entre el nitrógeno y fósforo sobre la producción de Astaxantina.

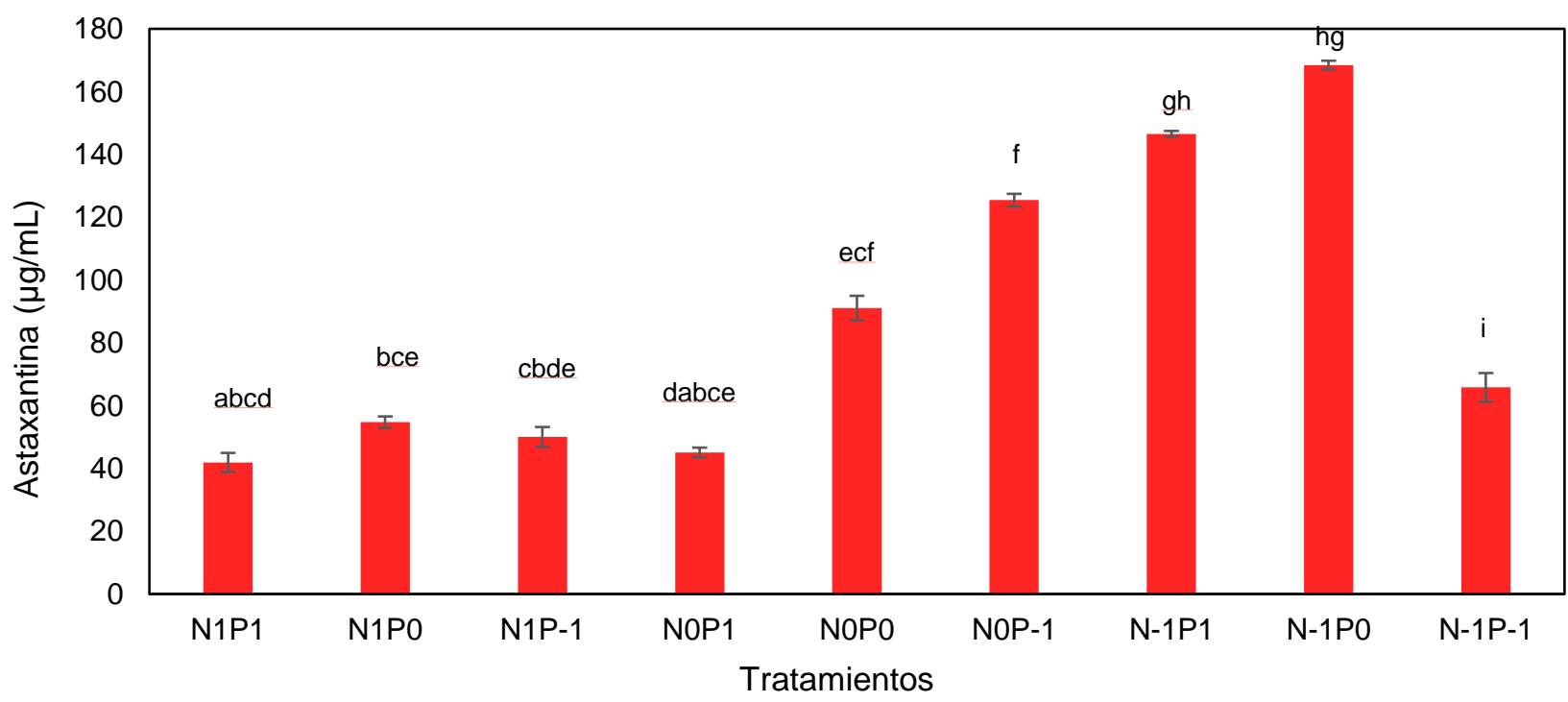

Fig. 3: Concentración de Astaxantina en $\mu \mathrm{g} / \mathrm{mL}$ obtenida de $H$. pluvialis UTEX 2505 en diferentes concentraciones de la fuente de nitrógeno y fósforo. Las letras iguales, corresponden a grupos estadísticamente homogéneos según el test de Tukey, con un nivel de confianza del $95 \%$.

Los ácidos grasos palmítico, nervonico, linolenico, araquidónico y linoleico se constituyen como los principales componentes del perfil lipídico, siendo los tres últimos de gran importancia en la nutrición humana. Además se debe considerar la importancia antioxidante del ácido linolenico. Cabe destacar que los principales ácidos grasos encontrados en la microalga son los reportados por diferentes autores como (Sanchez et al., 2012; Damiani et al., 2010; Miao et al., 2006), los cuales son usados por H.pluvialis en la esterificación de la Astaxantina ya sea en monoester o diester; brindándole a la célula una fuente de reserva energética. En condiciones de estrés (limitación de nitrógeno y fósforo) H.pluvialis UTEX 2505 presenta la capacidad de acumular ácidos grasos ya que se favorece la ruta metabólica del isoprenoide utilizada para la biosíntesis de Astaxantina (Chen et al., 2015). El ácido linoleico se presentó en mayor proporción en los diferentes tratamientos, como se observa en la figura 4. Por otra parte se determinó que en condiciones de limitación de nutrientes $\left(\mathrm{NaNO}_{3}, \mathrm{KH}_{2} \mathrm{PO}_{4}\right.$ y $\left.\mathrm{K}_{2} \mathrm{HPO}_{4}\right)$ el perfil lipídico de H.pluvialis UTEX 2505 es similar en cuanto a su composición en los diferentes tratamientos realizados, en el mismo grafico se denota el comportamiento de los lípidos totales, mostrando un máximo en el tratamiento de $\mathrm{N}_{0} \mathrm{P}_{0}$.

Por medio del coeficiente de correlación de Pearson se identificó la relación positiva moderada entre las variables dependientes Astaxantina y acumulación de lípidos, con un valor de 0.586 , lo que sugiere que las variables tienen una relación directa. Estudios anteriores reportan que aproximadamente el $95 \%$ de moléculas de Astaxantina se esterifican con ácidos grasos y se almacenan en cuerpos citosólicos ricos en triacilglicerol (Chen et al., 2015). 


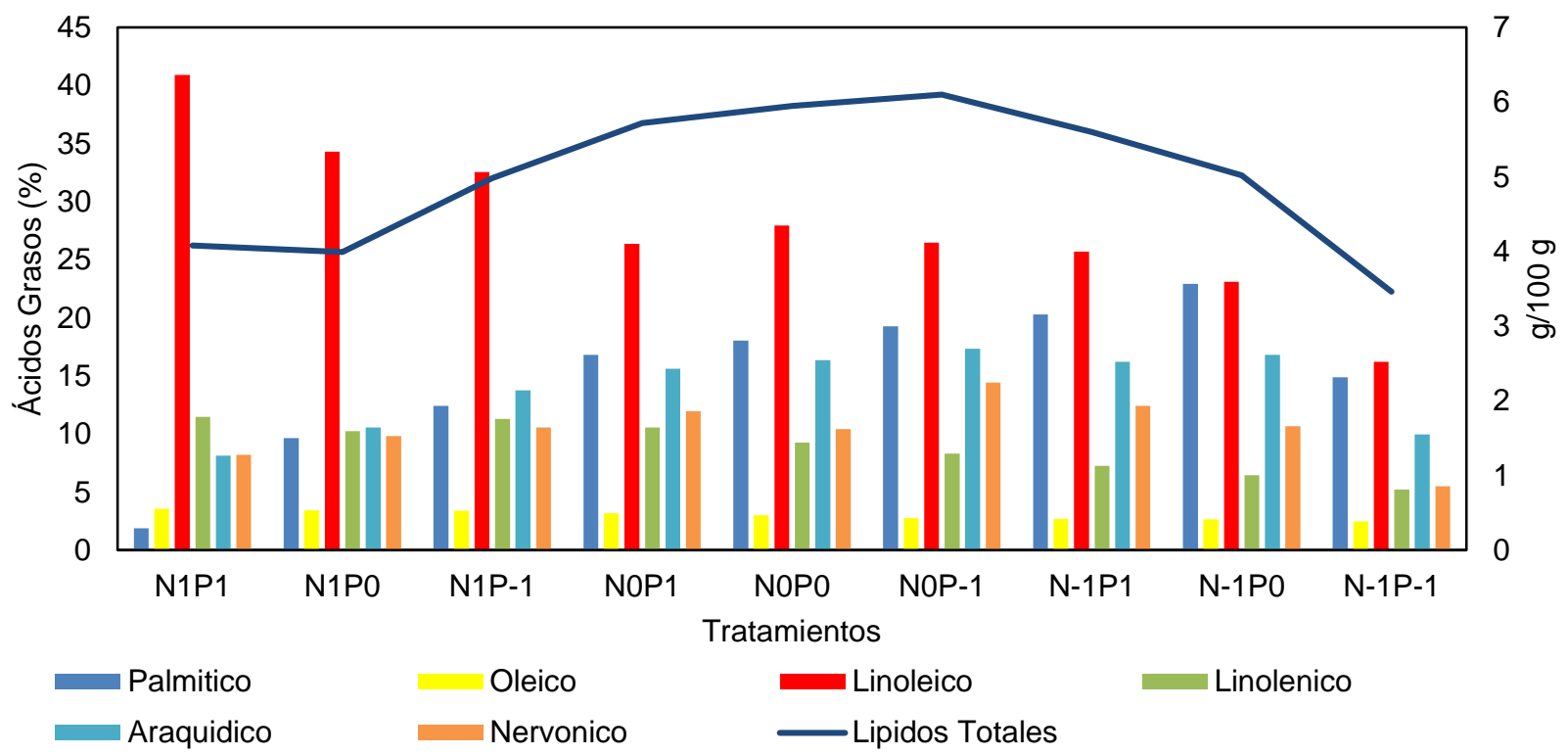

Fig. 4: Porcentaje de ácidos grasos en la fracción lipídica obtenida de H. pluvialis UTEX 2505 para cada tratamiento.

La interacción causada por los factores sobre el módulo de Young en H. pluvialis UTEX 2505 se presentan en la figura 5 , estableciendo que la interacción es negativa a niveles bajos de nitrógeno $(0.2 \mathrm{~g} / \mathrm{L})$. Posiblemente la baja concentración en la fuente de nitrógeno disminuye la rigidez en la pared celular de la microalga, y aumenta a niveles altos de nitrógeno $(1.0 \mathrm{~g} / \mathrm{L})$. Los módulos de Young para los diferentes tratamientos se muestran en la figura 6, donde a partir del análisis de varianza (ANOVA) se determinó que existe un efecto significativo sobre el módulo de Young por parte de la fuente de nitrógeno y la interacción de los factores en estudio, $(P<0.05)$ con un nivel de confianza del $95 \%$.

El tratamiento $\mathrm{N}_{0} \mathrm{P}_{1}$ presento el mayor módulo de Young de $469.81 \mathrm{MPa}$ y menor acumulación de Astaxantina $(45 \mu \mathrm{g} / \mathrm{mL})$, a diferencia del tratamiento $\mathrm{N}_{-1} \mathrm{P}_{0}$ que mostro menor valor $(2.92 \mathrm{MPa})$ de módulo de Young y mayor concentración de Astaxantina $(168 \mu \mathrm{g} / \mathrm{mL})$.Por lo tanto es posible asociar la rigidez que presenta $H$. pluvialis UTEX 2505 con la acumulación del carotenoide (Guerin et al., 2003), es decir, al generar estrés a la microalga por limitación de nutrientes (fuente de nitrógeno y fósforo) la rigidez de la célula disminuye debido a la presencia de Astaxantina en la membrana celular. Urnau et al. (2018) sugiere que la rigidez de la pared celular hace difícil la extracción de carotenoides. En lo que concierne a la acumulación de lípidos, se tiene que, a mayor concentración de ácidos grasos saturados, la rigidez es menor por lo que tanto los lípidos como la Astaxantina afectan el módulo de Young de H. pluvialis UTEX 2505.

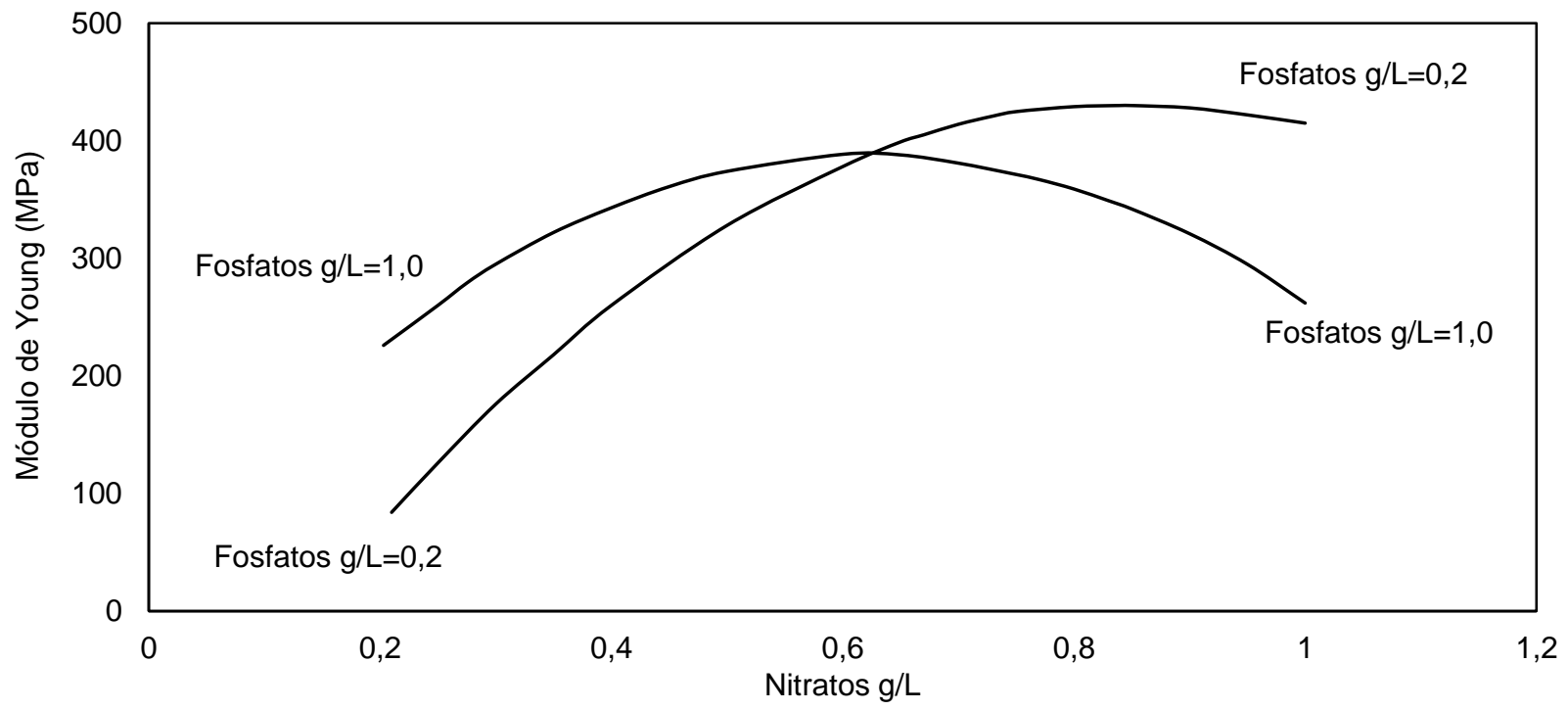

Fig. 5: Efecto de las interacciones entre el nitrógeno y fósforo sobre el módulo de elasticidad obtenido de H. pluvialis UTEX 2505. 


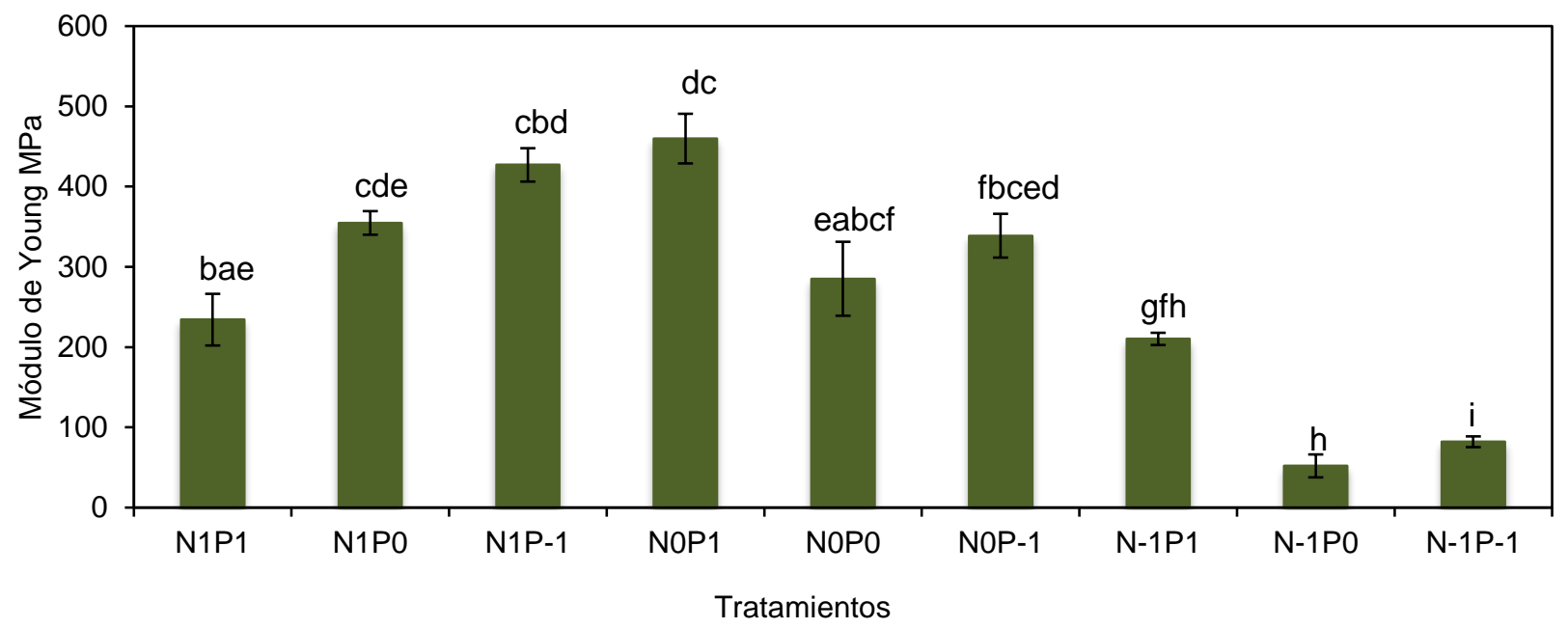

Fig. 6: Módulo de elasticidad obtenido de H. pluvialis UTEX 2505 en diferentes concentraciones de nitratos y fosfatos. Las letras iguales, corresponden a grupos estadísticamente homogéneos según el test de Tukey, con un nivel de confianza del 95\%.

\title{
CONCLUSIONES
}

De acuerdo a los resultados, análisis y discusión es posible extraer las siguientes conclusiones: 1) no se obtuvo efecto positivo por parte de la fuente de nitrógeno y fósforo en cuanto al crecimiento celular; 2) se encontró que las bajas concentraciones en la fuente de nitrógeno afecta positivamente la acumulación de Astaxantina en H.pluvialis UTEX 2505; 3) fue posible determinar el efecto positivo de la fuente de nitrógeno y fósforo en el perfil lipídico de la microalga. 4) se logró establecer que la rigidez de la membrana celular de la microalga es afectada negativamente por la acumulación de la Astaxantina.

\section{AGRADECIMIENTOS}

Agradecimientos a la universidad EAFIT - Colombia y al Centro de Argos para la Innovación por financiar la investigación.

\section{NOTACIÓN}

\author{
Abreviaciones \\ $\mathrm{AFM}=$ Microscopio de fuerza atómica \\ $\mathrm{CAPI}=$ Centro de Argos para la Innovación \\ $\mathrm{FBR}=$ Fotobiorreactores \\ $\mathrm{BBM}=$ Medio Basal Bold
}

\section{REFERENCIAS}

Anila, N., D. Simon, A. Chandrashekar, G. Ravishankar y R. Sarada, Metabolic Engineering of Dunaliella Salina for Production of Ketocarotenoids, Photosynthesis Research, 127(3), 321-333 (2016)

Borowitzka, M.A., J.M. Huisman y A. Osborn, Culture of the Astaxanthin-Producing Green alga Haematococcus Pluvialis 1. Effects of Nutrients on Growth and Cell Type, Journal of Applied Phycology, 3(4), 295-304 (1991)

Camacho, J.E., G. González y B. Klotz, Producción de Astaxantina en Haematococcus Pluvialis Bajo Diferentes Condiciones de Estrés, Nova, 11(19), 91-102 (2013)

Chen, G., B. Wang, D. Han, M. Sommerfeld, Y. Lu, F. Chen y Q. Hu, Molecular Mechanisms of the Coordination Between Astaxanthin and Fatty Acid Biosynthesis in Haematococcus Pluvialis (Chlorophyceae), Plant Journal, 81(1), 95-107 (2015)

Choi, Y.E., Y.S. Yun, J.M. Park y J.W. Yang, Determination of the Time Transferring Cells for Astaxanthin Production Considering Two-Stage Process of Haematococcus Pluvialis Cultivation, Bioresource Technology, 102(24), 11249-53 (2011)

Cifuentes, A.S., M.A. González, S. Vargas, M. Hoeneisen y N. González, Optimization of Biomass, Total Carotenoids and Astaxanthin Production in Haematococcus Pluvialis Flotow Strain Steptoe (Nevada, USA) Under Laboratory Conditions, Biological Research, 36(3-4), 343-57 (2003)

Colabella, F. y D. Libkind, PCR-Based Method for the Rapid Identification of Astaxanthin-Accumulating Yeasts (Phaffia Spp.), Revista Argentina de Microbiología, 48(1), 15-20 (2016)

Cömert, E.D. y V. Gökmen, Evolution of Food Antioxidants as a Core Topic of Food Science for a Century, Food Research International, 105, 76-93 (2018) 
Damiani, M.C., C.A. Popovich, D. Constenla y P.I. Leonardi, Lipid Analysis in Haematococcus Pluvialis to Assess Its Potential Use as a Biodiesel Feedstock, Bioresource Technology, 101(11), 3801-3807 (2010)

Galarza, J.I., J.A. Gimpel, V. Rojas, B.O. Arredondo y V. Henríquez, Over-Accumulation of Astaxanthin in Haematococcus Pluvialis Through Chloroplast Genetic Engineering, Algal Research, 31, 291-297 (2018)

Giannelli, L., H. Yamada, T. Katsuda y H. Yamaji, Effects of Temperature on the Astaxanthin Productivity and Light Harvesting Characteristics of the Green Alga Haematococcus Pluvialis, Journal of Bioscience and Bioengineering, 119(3), 345-350 (2015)

Guerin, M., M.E. Huntley y M. Olaizola, Haematococcus Astaxanthin:Applications for Human Health and Nutrition, Trends Biotechnology, 21(5), 210-216 (2003)

Hernández, K.J., M.E. Perez y otros tres autores, Haematococcus Pluvialis, Revisión Bibliográfica 2003-2013, Rev. Mex. Cien. Farm., 46(1), 7-16 (2015)

Higuera, I., L. Félix y F. M. Goycoolea, Astaxanthin: A Review of Its Chemistry and Applications, Critical Reviews in Food Science and Nutrition, 46(2), 185-196 (2006)

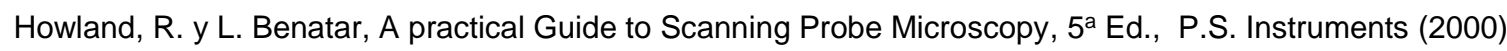

Justo, C. y R.V. Gutiérrez, Daño Oxidativo, Radicales Libres y Antioxidantes, Rev. Cubana Med. Milit., 31(2), 126-133 (2002)

Li, Y.X., F.J. Zhao y D.D. Yu, Effect of Nitrogen Limitation on Cell Growth, Lipid Accumulation and Gene Expression in Chlorella sorokiniana, Brazilian Archives of Biology and Technology, 58(3), 462-467 (2015)

Liu, J., Z. Sun y otros cuatro autores, Chlorella Zofingiensis As an Alternative Microalgal Producer of Astaxanthin: Biology and Industrial Potential, Marine drugs, 12(6), 3487-3515 (2014)

Maldonado, O., E.N. Jiménez y otros tres autores, Radicales Libres y su Papel en Las Enfermedades CrónicoDegenerativas, Revista Médica de La Universidad Veracruzana, (272), 32-39 (2010)

Miao, F., D. Lu, Y. Li y M. Zeng, Characterization of Astaxanthin Esters in Haematococcus Pluvialis by Liquid Chromatography-Atmospheric Pressure Chemical Ionization Mass Spectrometry, Analytical Biochemistry, 352(2), 176$181(2006)$

Orosa, M., D. Franqueira, A. Cid y J. Abalde, Analysis and Enhancement of Astaxanthin Accumulation in Haematococcus Pluvialis, Bioresource Technology, 96(3), 373-378 (2005)

San-Miguel, A. y F.J. Martin, Importancia de Las Especies Reactivas Al Oxigeno (Radicales Libres) y los Antioxidantes En Clinica, Gaceta Médica de Bilbao, 106(3), 106-113 (2009)

Sánchez, A.P., M.A. Meireles, A.L. Ferreira, E. Saito y F.A. Cabral, Extraction of $\omega-3$ Fatty Acids and Astaxanthin from Brazilian Redspotted Shrimp Waste Using Supercritical $\mathrm{CO}_{2}$ + Ethanol Mixtures, The Journal of Supercritical Fluids, 61, 71-77 (2012)

Shah, M.M., Y. Liang, J.J. Cheng y M. Daroch, Astaxanthin-Producing Green Microalga Haematococcus Pluvialis: From Single Cell to High Value Commercial Products, Frontiers in Plant Science, 7, 1-28 (2016)

Suh, I.S., H.N. Joo, C.G. Lee, A Novel Double-Layered Photobioreactor for Simultaneous Haematococcus Pluvialis Cell Growth and Astaxanthin Accumulation, J. Biotechnol, 125(4), 540-546 (2006)

Urnau, L., R. Colet y otros cuatro autores, Extraction of Caretenoids from Xanthophyllomyces dendrorhous Using Ultrasound-Assisted and Chemical Cell Disruption Methods, The Canadian Journal of Chemical Engineering, 96(6), 1377$1381(2018)$

Valko, M., D. Leibfritz y otros cuatro autores, Free Radicals and Antioxidants in Normal Physiological Functions and Human Disease, The International Journal of Biochemistry \& Cell Biology, 39(1), 44-84 (2007)

Warren, K.M., J.N. Mpagazehe, P.R. LeDuc y C.F. Higgs III, Probing the Elastic Response of Microalga Scenedesmus Dimorphus in Dry and Aqueous Environments Through Atomic Force Microscopy, Applied Physics Letters, 105 (2014)

Yamashita, E., Astaxanthin as a Medical Food, Functional Foods in Health and Disease, 3(7), 254-258 (2013)

Zhang, B.Y., Y.H. Geng. Z.K. Li, H.J. Hu e Y.G. Li, Production of Astaxanthin From Haematococcus in Open Pond by TwoStage Growth One-Step Process, Aquaculture, 295(3-4), 275-281 (2009)

Zhang, W., J. Wang, J. Wang y T. Liu, Attached Cultivation of Haematococcus Pluvialis for Astaxanthin Production, Bioresource Technology, 158, 329-335 (2014)

Zheng, Y.G., Z. Wang e Y. Shen, Large-Scale Production of Astaxanthin by Xanthophyllomyces Dendrorhous, Food and Bioproducts Processing, 84(2), 164-166 (2006)

Zuluaga, M., V. Gueguen, D. Letourneur y G. Pavon, Astaxanthin-Antioxidant Impact on Excessive Reactive Oxygen Species Generation Induced by Ischemia and Reperfusion Injury, Chemico-Biological Interactions, 279, 145-158 (2018) 
